\title{
Nonlinear cavity feeding and unconventional photon statistics in solid-state cavity QED revealed by many-level real-time path-integral calculations
}

\author{
M. Cygorek, ${ }^{1}$ A. M. Barth,${ }^{1}$ F. Ungar, ${ }^{1}$ A. Vagov, ${ }^{1}$ and V. M. Axt ${ }^{1}$ \\ ${ }^{1}$ Theoretische Physik III, Universität Bayreuth, 95440 Bayreuth, Germany
}

\begin{abstract}
The generation of photons in a microcavity coupled to a laser-driven quantum dot interacting with longitudinal acoustic (LA) phonons is studied in the regime of simultaneously strong driving and strong dot-cavity coupling. The stationary cavity photon number is found to depend in a nontrivial way on the detuning between the laser and the exciton transition in the dot. In particular, the maximal efficiency of the cavity feeding is obtained for detunings corresponding to transition energies between cavity-dressed states with excitation numbers larger than one. Phonons significantly enhance the cavity feeding at large detunings. In the strong-driving, strong-coupling limit, the photon statistics is highly non-Poissonian. While without phonons a double-peaked structure in the photon distribution is predicted, phonons make the photon statistics thermal-like with very high effective temperatures $\sim 10^{5} \mathrm{~K}$, even for low phonon temperatures $\sim 4 \mathrm{~K}$. These results were obtained by numerical calculations where the driving, the dot-cavity coupling and the dot-phonon interactions are taken into account without approximations. This is achieved by a reformulation of an exact iterative path-integral scheme which is applicable for a large class of quantum-dissipative systems and which in our case reduces the numerical demands by 15 orders of magnitude.
\end{abstract}

Solid-state quantum dots (QDs) have attracted much attention in recent years since they promise applications in photonic devices and quantum information technology, e.g., as qubits [1] and sources of single [2-4] or entangled photons [5-7]. Embedding QDs in microcavities increases the light extraction efficiency via the Purcell effect [2] and allows one to study cavity QED in solid-state systems [8-16]. Cavities may be used as busses mediating a selective coupling of two qubits stored in two QDs within the same cavity [17-19]. Investigating cavity QED in a solid is a rich field, also because the interaction with LA phonons may have a profound impact on the physics [20-22], e.g., enabling phonon-mediated inversion of the QD [23] or providing a non-resonant coupling between $\mathrm{QD}$ and cavity [24].

Recently, experiments [25] on solid-state cavity QED systems have advanced into the largely unexplored regime of strong dot-cavity coupling combined with strong laser driving. While strongly driven dots can be described in terms of laser-dressed states and the physics of strongly coupled dot-cavity systems is best discussed in the basis of the cavity-dressed states, developing a physical intuition is more difficult when driving and coupling are equally strong. A further challenge is the coupling of the QD to phonons, in particular since phonon influences on the QD dynamics are typically not well described by Born-Markov rate equations [26]. Thus, more sophisticated approaches are required, like the correlation expansion [27-29] or polaron master equations [14, 22, 30-32].

For possible applications of strongly coupled dot-cavity systems, e.g., as building blocks of photonic devices, it is of fundamental interest how efficiently photons can be generated in the microcavity by driving the QD with an external laser field. In this letter, we address this question for cases where QD-cavity and QD-laser couplings are simultaneously strong while also accounting

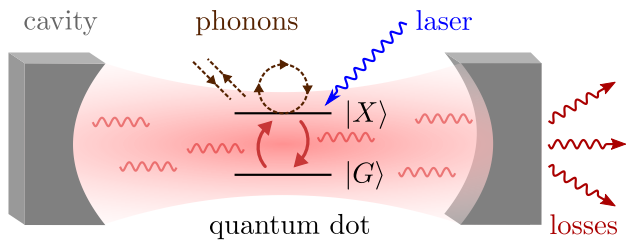

FIG. 1. Laser-driven quantum dot inside a microcavity and coupled to longitudinal acoustic phonons.

for the QD-phonon interaction. Intuition suggests that the cavity feeding is enhanced when the laser is tuned to the resonances in the linear absorption of the dotcavity system, i.e., when the dot-laser detuning matches the vacuum-Rabi-split peaks corresponding to the transitions between the ground state and the first excited cavity-dressed states. We demonstrate that this expectation is only confirmed at low driving strength where the average cavity photon number is much smaller than one due to the photon blockade [33]. At strong driving, we find the maximum feeding efficiency at much smaller detunings, while there is no enhancement at the resonances of the linear absorption.

Phonons are a major reason why solid-state cavity QED differs from atomic cavity QED. In most cases phonons limit the performance of device relevant processes by introducing decoherence. However, in this letter we demonstrate that, already for small detunings compared to the vacuum Rabi splitting, phonons lead to a strongly enhanced generation of cavity photons that becomes almost independent of the detuning and all traces of the photon blockade are eliminated.

While in weak-coupling or weak-driving situations only cavity states with one or two photons can be significantly occupied, strong-driving+strong-coupling conditions lead to the excitation of states with larger photon 
numbers. This makes the shape of the photon distribution a meaningful target for investigations. In contrast to the case of classical (direct) driving of a cavity, which leads to a Poissonian photon distribution [34], we find strongly non-Poissonian distributions when the cavity is driven indirectly via the dot. Here, phonons not only lead to drastic quantitative effects but change the photon statistics qualitatively by transforming the photon distribution into a nearly thermal one.

We study a QD-cavity system interacting with LA phonons considering a single ground-to-exciton transition of the QD coupled to a single cavity mode and driven by an external laser field as sketched in Fig. 1. The Hamiltonian for the dot-cavity system in the interaction picture with respect to the laser energy $\hbar \omega_{L}$ is given by

$$
\begin{aligned}
H_{N}= & \hbar \Delta \omega_{X L}|X\rangle\langle X|-\hbar f(|G\rangle\langle X|+| X\rangle\langle G|) \\
& +\hbar \Delta \omega_{c L} \hat{a}^{\dagger} \hat{a}+\hbar g\left(\hat{a}^{\dagger}|G\rangle\langle X|+\hat{a}| X\rangle\langle G|\right),
\end{aligned}
$$

where $|G\rangle(|X\rangle)$ denotes the ground (excited) state of the $\mathrm{QD}$ and $\hat{a}^{\dagger}(\hat{a})$ is the creation (annihilation) operator of a cavity photon. $\hbar \Delta \omega_{X L}=\hbar\left(\omega_{X}-\omega_{L}\right)$ and $\hbar \omega_{X}$ is the exciton transition energy while $\hbar \Delta \omega_{c L}=\hbar \omega_{c}-\hbar \omega_{L}$ is the detuning of the cavity with respect to the external driving. $g$ is the strength of the dot-cavity coupling and $f$ denotes the strength of the external $\mathrm{cw}$ laser driving.

LA phonons are included by the Hamiltonian [35, 36]

$$
\hat{H}_{p h}=\hbar \sum_{\mathbf{q}} \omega_{\mathbf{q}} \hat{b}_{\mathbf{q}}^{\dagger} \hat{b}_{\mathbf{q}}+\hbar \sum_{\mathbf{q}}\left(\gamma_{\mathbf{q}}^{X} \hat{b}_{\mathbf{q}}^{\dagger}+\gamma_{\mathbf{q}}^{X * \hat{b}_{\mathbf{q}}}\right)|X\rangle\langle X|,
$$

where $\hat{b}_{\mathbf{q}}^{\dagger}$ and $\hat{b}_{\mathbf{q}}$ are creation and annihilation operators for phonons with energy $\hbar \omega_{\mathbf{q}} \cdot \gamma_{\mathbf{q}}^{X}$ are the excitonphonon-coupling matrix elements given explicitly in the supplement. Finally, cavity losses are taken into account via the Lindblad term $\mathcal{L}_{\text {loss }}[\hat{\rho}]=\kappa\left[\hat{a} \hat{\rho} \hat{a}^{\dagger}-\frac{1}{2}\left\{\hat{\rho}, \hat{a}^{\dagger} \hat{a}\right\}_{+}\right]$, with loss rate $\kappa$.

The number of cavity photons generated by driving via the dot is limited by the losses. However, when the driving $(f \gg \kappa)$ and the QD-cavity coupling $(g \gg \kappa)$ are strong, describing the dynamics requires accounting for a large number of states of the QD-cavity system. Here we consider all dot-cavity product states with up to $n_{x} \leq 20$ excitations, where the excitation number $n_{x}$ is the photon number $n$ plus the exciton occupation. Since there is one pair of states $\left(\left|G, n_{x}\right\rangle,\left|X, n_{x}-1\right\rangle\right)$ per excitation $n_{x}>0$ and only one state $|G, 0\rangle$ with $n_{x}=0$, this amounts to a system with $N=41$ levels.

It is important to note that strongly-driven, stronglycoupled solid-state cavity QED systems are simultaneously highly non-linear with respect to the driving, the dot-cavity coupling, and the dot-phonon coupling, so that there is no obvious small parameter in the system. As a consequence, it is a priory unclear whether conclusive results can be obtained from established approximate methods, such as master equation or correlation expansion approaches. In principle, the time evolution of the reduced density matrix of an $N$-level system coupled to a continuum of phonons can be calculated using numerically exact path integral (PI) methods [20, 37-40]. Unfortunately, the numerical effort required in such calculations rises exponentially with the number of levels $N$, so that complete PI simulations have been performed only for rather small systems [20, 41-46]. In particular, when the phonon-induced memory time is as long as a few picoseconds (which is typical for LA phonons coupled to a QD) numerically complete simulations are usually restricted to $N \leq 4$, while larger systems may be treated by heuristically discarding a large number of paths with numerically small contributions [47-49]. For solid-state cavity QED systems with $N \sim 40$ levels, complete PI simulations have, so far, been performed only for lossless cavities without external driving [50], where the quantum dynamics of the system mixes only pairs of states with fixed excitation numbers $n_{x}$. However, external driving or cavity losses introduce transitions between states with different excitation numbers, so that the fully coupled system has to be considered, which increases the computation time dramatically.

We have overcome this problem by reformulating a standard iterative algorithm [37] to perform the sum over the paths, which in our case reduces the number of entities that have to be iterated by more than 15 orders of magnitude without introducing approximations. Full details of the reformulated path-integral method and its derivation are given in the supplement. Here, we only note that the reformulation can be applied generally to any $N$-level system coupled to an oscillator continuum, provided the $N$ states can be subdivided into $N_{g}$ groups with identical oscillator couplings within each group. In our case, the interaction with phonons does not distinguish between states that differ only in the number of cavity photons. Thus, we have $N_{g}=2$ were one group comprises the states $\left|G, n_{x}\right\rangle$ while a second group contains the states $\left|X, n_{x}-1\right\rangle$.

It is worthwhile to note that apart from the specific case treated in this letter there is a wealth of other systems of topical interest where the new algorithm can be applied. An example of such systems are QDs with embedded magnetic dopants, e.g., Mn ions, which are highly attractive for spintronic applications [51-53]. Here, the phonon coupling does not distinguish between different spin configurations of the dot-dopant system. Another possible application is the description of phonon effects on the biexciton cascade in a $\mathrm{QD}$, proposed as a source of entangled photon pairs [5-7]. As in our system, the phonon coupling does not distinguish between states differing only by photon numbers such that for the biexciton cascade $N_{g}=3$ groups have to be considered (ground state, excitons, and biexciton). Cavity QED has also been studied in systems where superconducting charge qubits are strongly coupled to a microwave cavity [54]. 

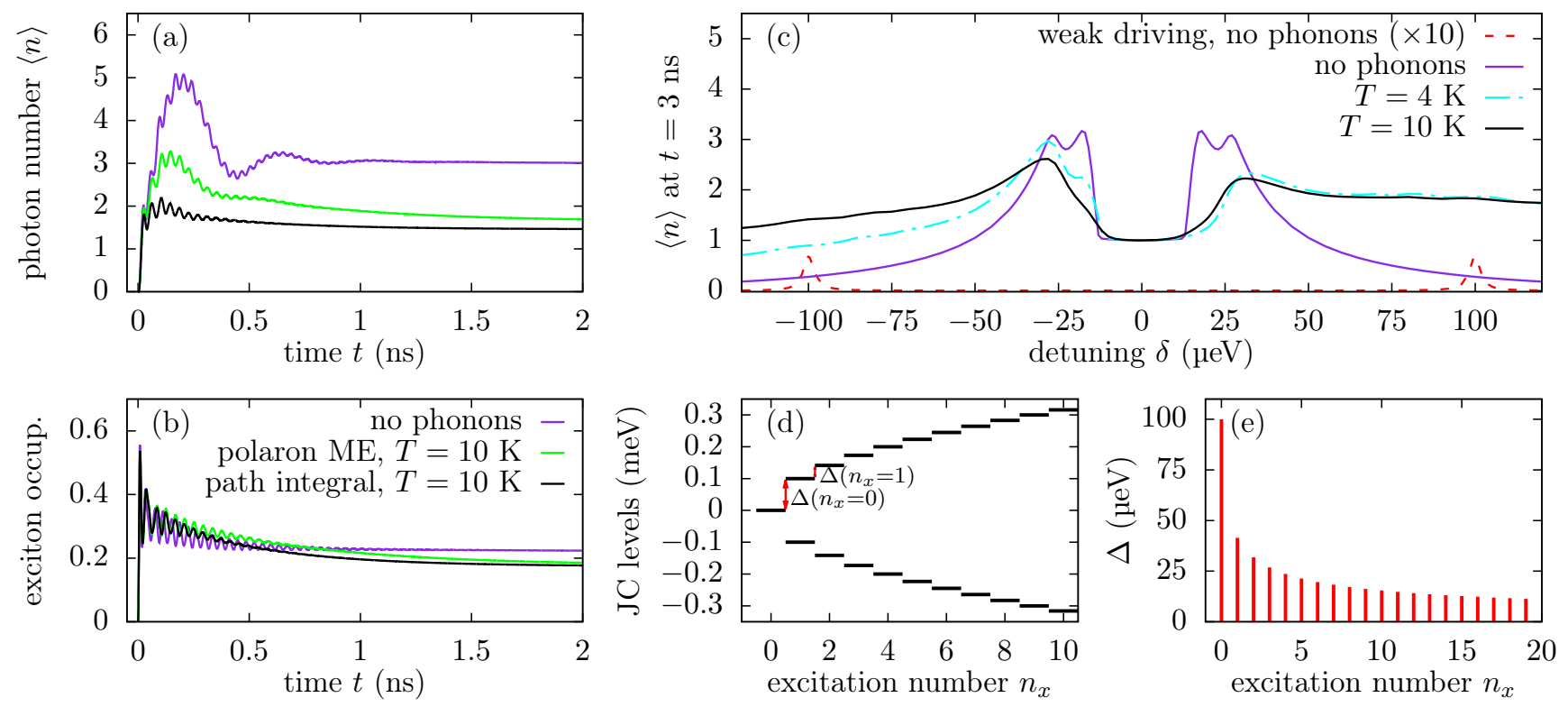

FIG. 2. Time evolution of the average photon number (a) and of the exciton occupation (b) for a detuning $\delta=20 \mu \mathrm{eV}$ at $T=10 \mathrm{~K}$ calculated using the path-integral theory (black), Markovian polaron master equations (green), and without phonons (violet). (c): Average photon number $\langle n\rangle$ in the cavity at $t=3 \mathrm{~ns}$ as a function of the detuning $\delta$. (d): Energy of the Jaynes-Cummings levels in the rotating frame. (e): Transition energies $\Delta\left(n_{x}\right)$ between neighboring Jaynes-Cummings levels [as indicated in $(\mathrm{d})]$.

There, the dephasing is determined by charge fluctuations that can be represented by a bath of harmonic oscillators [55], so that our PI method can be applied to these systems as well.

For the present study of cavity feeding in QD-based solid-state cavity QED systems, we assume a cavity in resonance with the polaron-shifted QD-exciton transition and a laser coupled to the QD transition detuned by an energy $\delta$ from the cavity mode. Before the laser is switched on at time $t=0$ the electronic system is in the ground state $|G, 0\rangle$ while the phonons are initially in thermal equilibrium at temperature $T$. The dot-cavity coupling and the driving are chosen to be of equal strength $\hbar g=\hbar f=0.1 \mathrm{meV}$, if not stated otherwise. The cavity loss rate is taken to be $\kappa=0.01 \mathrm{ps}^{-1}$, which corresponds to a quality factor $Q \approx 10^{5}$. For the phonon environment and dot-phonon coupling we assume parameters of a selfassembled InGaAs QD with radius $a_{e}=3 \mathrm{~nm}$ embedded in a GaAs matrix (cf. supplement).

Figures 2(a) and (b) depict the time evolution of the average cavity photon number $\langle n\rangle$ and the exciton occupation for a detuning $\delta=20 \mu \mathrm{eV}$. Both quantities show an oscillatory transient behavior in the first $\sim 1 \mathrm{~ns}$ and eventually reach stationary values. We find that phonons have little impact on the exciton occupations but can change the average photon number significantly. For comparison, we present results obtained using polaron master equations (PMEs, cf. supplement), a well established method for the treatment of the dot-phonon inter- action $[14,30,32,56]$. These results demonstrate that, while exciton occupations are reasonably well described by the PMEs, photon related quantities are much more affected by the approximations used to derive the PMEs. In particular, the PMEs predict significantly larger (e.g., more than $60 \%$ at $t=150 \mathrm{ps}$ ) photon numbers than the PI method, revealing that the PMEs are insufficient for an accurate description of the situation studied here. We note that this inaccuracy of the PMEs can be explained by the fact that the dot-cavity system possesses a number of different energy gaps on scales $\sim 10-1000 \mu \mathrm{eV}$ [cf. Fig. 2(d)], which can be bridged by phonon-assisted transitions. These are, however, only strictly treated up to second order in the dot-phonon interaction within the PME approach.

Phonon effects on the cavity feeding are illustrated in Fig. 2(c) showing the average photon number at long times $(t=3 \mathrm{~ns})$ as a function of $\delta$ with and without dotphonon interaction. In the weak-driving limit ( $\hbar f=1$ $\mu \mathrm{eV}$, red line), the driving of the cavity via the dot is most effective if one excites at the resonances of the linear absorption, manifested in the appearance of two peaks at $\delta= \pm \hbar g= \pm 100 \mu \mathrm{eV}$ separated by the vacuum Rabi splitting. Surprisingly, for strong driving the maximum number of photons in the cavity is obtained neither at $\delta= \pm \hbar g$ nor when the driving is in resonance with the ground-to-exciton transition $(\delta=0$, i.e., where the linear absorption has a resonance for vanishing dot-cavity coupling), but in a region with small detunings of $\sim 20-40$ 


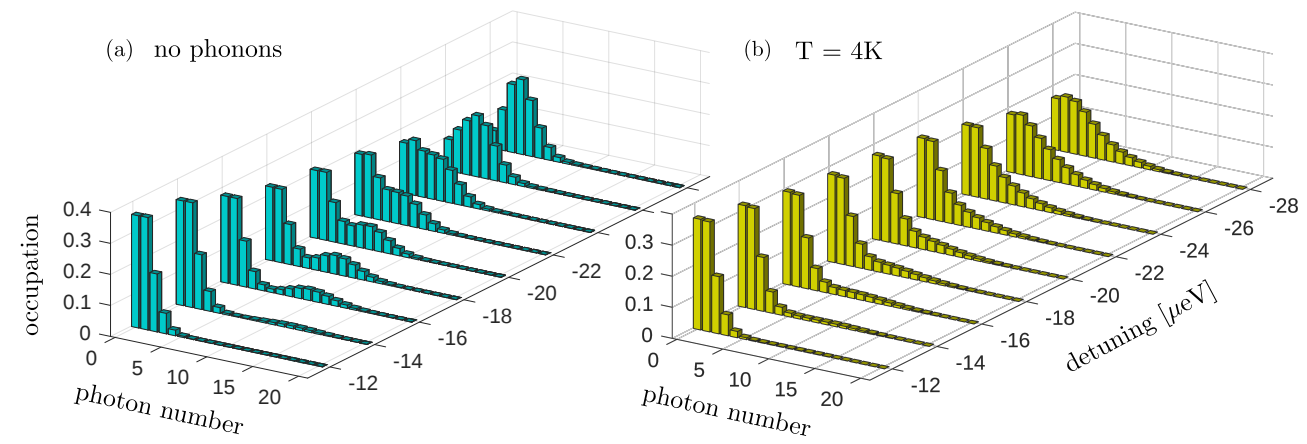

(c) detuning $=-18 \mu \mathrm{eV}$

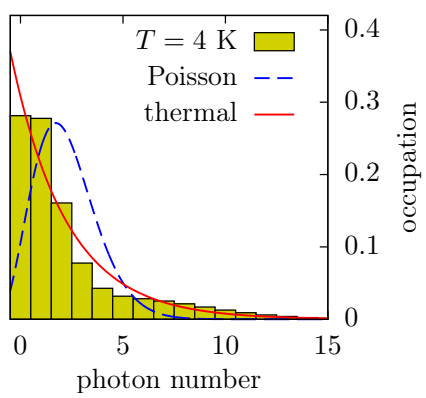

FIG. 3. Cavity photon distribution at $t=3 \mathrm{~ns}$ for different detunings $\delta$ and $\hbar g=\hbar f=0.1$ meV without dot-phonon interaction (a) and with phonons at temperature $T=4 \mathrm{~K}(\mathrm{~b})$. (c): Photon distribution at detuning $\delta=-18 \mu \mathrm{eV}$ with phonons at $T=4$ $\mathrm{K}$ compared with Poissonian and thermal distributions.

$\mu \mathrm{eV}$. This is explained by the fact that with strong driving the cavity is partially filled with photons and thus transitions between states with higher photon numbers become important. When the dot and the cavity are in resonance, the energy eigenvalues of the cavity-dressed states are $E_{n_{x}}= \pm \sqrt{n_{x}} \hbar g$ [cf. Fig 2(d)] and transitions between neighboring states can occur at energies $\Delta\left(n_{x}\right)=\left(\sqrt{n_{x}+1}-\sqrt{n_{x}}\right) \hbar g$ depicted in Fig. 2(e). For the chosen parameters, the efficiency of the cavity feeding increases drastically when $\delta$ becomes similar to $\Delta\left(n_{x}\right)$ for $n_{x} \approx 5$, which corresponds to the typical photon number of states that are significantly occupied. When the driving strength increases further and states with higher photon numbers are occupied, the maxima in Fig 2(c) will eventually shift to zero and merge into the central peak of a Mollow triplet [57].

Fig. 2(c) also reveals an asymmetry of the average photon number with respect to a sign change of the detuning. This asymmetry diminishes at higher temperatures indicating that it originates from the asymmetry between phonon absorption and emission. Compared with phonon-free calculations, the interaction with phonons results in a reduced efficiency at the maxima because it suppresses the coherent driving. However, at larger detunings, phonon-assisted processes facilitate otherwise prohibited transitions between off-resonant states, which enables a much more efficient generation of cavity photons. Interestingly, the phonon-induced feeding efficiency in this regime is almost independent of the detuning in a wide parameter range.

More detailed information than the mean number of photons is provided by examining how the photons are distributed across different photonic states. It is well known that direct (classical) driving of a cavity leads to a Poissonian statistics [34]. Here, however, we find that driving the cavity via the dot creates highly nonPoissonian distributions as depicted in Fig. 3. Figure 3(a) illustrates the case where the dot-phonon interaction is neglected. There, large detunings lead to a shift of the peak in the photon distribution to higher photon num- bers with a rapidly decaying tail, as expected for a Poisson distribution. For values of the detuning at which the resonance condition with neighboring cavity-dressed energy eigenstates is met, a double-peak structure appears in the photon statistics. This two-peak structure is a rather unconventional feature, which is possible only due to the resonance in the non-linear driving regime.

When the dot-phonon interaction is accounted for [cf. Fig. 3(b)], significant changes in the photon distributions are observed. Most prominently, the double-peak structure predicted by the phonon-free calculation disappears. Furthermore, for large photon numbers, the photon distribution has a maximum closer to $n=0$ and possesses a significantly longer tail than expected for a Poissonian distribution. In Fig. 3(c), we compare the photon statistics for $\delta=-18 \mu \mathrm{eV}$, where the maximal efficiency of cavity feeding was reached, with a Poissonian as well as with a thermal distribution $\left[P_{\operatorname{th}}(n)=\left(1-e^{-\epsilon}\right) e^{-\epsilon n}\right]$ with the same average photon number $\langle n\rangle \approx 2.25$ as obtained in the numerical simulations for $T=4 \mathrm{~K}$. It can be seen that the obtained photon statistics in the presence of phonons is much closer to the thermal distribution than to the Poissonian. For a cavity mode with energy $\hbar \omega_{c} \approx 1.5 \mathrm{eV}$, one can extract an effective temperature $T=\hbar \omega_{c} /\left(k_{B} \epsilon\right) \approx 47000 \mathrm{~K}$. It is worth noting that, although the qualitative change of the photon statistics toward a thermal distribution is caused by the dot-phonon interaction, the value of the photon temperature is four orders of magnitude larger than the phonon temperature and is rather determined by the average photon number, which can be tuned by changing the driving strength.

To summarize, we have investigated the generation of photons in a QD-cavity system coupled to LA phonons in the regime of strong driving and simultaneously strong coupling. Conclusive results have been obtained using a novel variant of the numerically exact real-time pathintegral approach, which for a large class of systems of topical interest speeds up the numerics by many orders of magnitude. Our simulations show that, when a dot is in resonance with a microcavity, the feeding efficiency 
depends non-trivially on the dot-laser detuning. In the strong-driving limit, the maximal feeding is observed for much smaller detunings than expected from the linear absorption. The dot-phonon interaction is found to suppress the feeding efficiency at resonances. However, for larger detunings, it opens up the possibility of a highly efficient phonon-mediated feeding which is robust against variations of the detuning. Furthermore, the dot-phonon interaction modifies the photon statistics qualitatively, so that a double peaked distribution for the phonon-free case is transformed to a nearly thermal occupation of photon states with a huge effective temperature.

We gratefully acknowledge the financial support from Deutsche Forschungsgemeinschaft via the Project No. AX 17/7-1.

[1] P. Chen, C. Piermarocchi, and L. J. Sham, Phys. Rev. Lett. 87, 067401 (2001).

[2] N. Somaschi, V. Giesz, L. De Santis, J. Loredo, M. P. Almeida, G. Hornecker, S. L. Portalupi, T. Grange, C. Antón, J. Demory, C. Gómez, I. Sagnes, N. D. Lanzillotti-Kimura, A. Lemaítre, A. Auffeves, A. G. White, L. Lanco, and P. Senellart, Nat. Photon. 10, 340 (2016).

[3] P. Michler, A. Kiraz, C. Becher, W. V. Schoenfeld, P. M. Petroff, L. Zhang, E. Hu, and A. Imamoglu, Science 290, 2282 (2000).

[4] Y.-M. He, Y. He, Y.-J. Wei, D. Wu, M. Atatüre, C. Schneider, S. Höfling, M. Kamp, C.-Y. Lu, and J.-W. Pan, Nat. Nanotechnol. 8, 213 (2013).

[5] M. A. M. Versteegh, M. E. Reimer, K. D. Jöns, D. Dalacu, P. J. Poole, A. Gulinatti, A. Guidice, and V. Zwiller, Nat. Commun. 5, 5298 (2014).

[6] M. Müller, S. Bounouar, K. D. Jöns, M. Glässl, and P. Michler, Nat. Photon. 8, 224 (2014).

[7] N. Akopian, N. H. Lindner, E. Poem, Y. Berlatzky, J. Avron, D. Gershoni, B. D. Gerardot, and P. M. Petroff, Phys. Rev. Lett. 96, 130501 (2006).

[8] D. Englund, A. Faraon, I. Fushman, N. Stoltz, P. Petroff, and J. Vučković, Nature Letters 450, 857 (2007).

[9] K. Hennessy, A. Badolato, M. Winger, D. Gerace, M. Atatüre, S. Gulde, S. Fält, E. L. Hu, and A. Imamoğu, Nature 445, 896 (2007).

[10] J. P. Reithmaier, Semicond. Sci. Technol. 23, 123001 (2008).

[11] J. Kasprzak, S. Reitzenstein, E. A. Muljarov, C. Kistner, C. Schneider, M. Strauss, S. Hfling, A. Forchel, and W. Langbein, Nat. Mater. 9, 304 (2010).

[12] S. Reitzenstein, IEEE Journal of Selected Topics in Quantum Electronics 18, 1733 (2012).

[13] J. Kasprzak, K. Sivalertporn, F. Albert, C. Schneider, S. Höfling, M. Kamp, A. Forchel, S. Reitzenstein, E. A. Muljarov, and W. Langbein, New Journal of Physics 15, 045013 (2013).

[14] C. Roy and S. Hughes, Phys. Rev. X 1, 021009 (2011).

[15] C. Roy and S. Hughes, Phys. Rev. Lett. 106, 247403 (2011).

[16] S. M. Ulrich, S. Ates, S. Reitzenstein, A. Löffler,
A. Forchel, and P. Michler, Phys. Rev. Lett. 106, 247402 (2011).

[17] M. Feng, I. D'Amico, P. Zanardi, and F. Rossi, Phys. Rev. A 67, 014306 (2003).

[18] A. Imamoglu, D. D. Awschalom, G. Burkard, D. P. DiVincenzo, D. Loss, M. Sherwin, and A. Small, Phys. Rev. Lett. 83, 4204 (1999).

[19] M. S. Sherwin, A. Imamoglu, and T. Montroy, Phys. Rev. A 60, 3508 (1999).

[20] D. G. Nahri, F. H. A. Mathkoor, and C. H. R. Ooi, Journal of Physics: Condensed Matter 29, 055701 (2017).

[21] P. Kaer, T. R. Nielsen, P. Lodahl, A.-P. Jauho, and J. Mørk, Phys. Rev. Lett. 104, 157401 (2010).

[22] P. Kaer, T. R. Nielsen, P. Lodahl, A.-P. Jauho, and J. Mørk, Phys. Rev. B 86, 085302 (2012).

[23] S. Hughes and H. J. Carmichael, New Journal of Physics 15, 053039 (2013).

[24] S. Ates, S. M. Ulrich, A. Ulhaq, S. Reitzenstein, A. Löffler, S. Höfling, A. Forchel, and P. Michler, Nature Photonics 3, 724 (2009).

[25] C. Hopfmann, A. Carmele, A. Musiał, C. Schneider, M. Kamp, S. Höfling, A. Knorr, and S. Reitzenstein, Phys. Rev. B 95, 035302 (2017).

[26] D. P. S. McCutcheon, N. S. Dattani, E. M. Gauger, B. W. Lovett, and A. Nazir, Phys. Rev. B 84, 081305 (2011).

[27] J. Förstner, C. Weber, J. Danckwerts, and A. Knorr, Phys. Rev. Lett. 91, 127401 (2003).

[28] M. Glässl, A. Vagov, S. Lüker, D. E. Reiter, M. D. Croitoru, P. Machnikowski, V. M. Axt, and T. Kuhn, Phys. Rev. B 84, 195311 (2011).

[29] D. E. Reiter, Phys. Rev. B 95, 125308 (2017).

[30] A. Nazir and D. P. S. McCutcheon, Journal of Physics: Condensed Matter 28, 103002 (2016).

[31] P. Karwat and P. Machnikowski, Phys. Rev. B 91, 125428 (2015).

[32] J. Iles-Smith and A. Nazir, Optica 3, 207 (2016).

[33] A. Faraon, I. Fushman, D. Englund, N. Stoltz, P. Petroff, and J. Vučković, Nature Physics 4, 859 (2008).

[34] R. J. Glauber, Phys. Rev. 131, 2766 (1963).

[35] L. Besombes, K. Kheng, L. Marsal, and H. Mariette, Phys. Rev. B 63, 155307 (2001).

[36] P. Machnikowski and L. Jacak, Phys. Rev. B 69, 193302 (2004).

[37] N. Makri and D. E. Makarov, The Journal of Chemical Physics 102, 4600 (1995).

[38] N. Makri and D. E. Makarov, The Journal of Chemical Physics 102, 4611 (1995).

[39] A. Vagov, M. D. Croitoru, M. Glässl, V. M. Axt, and T. Kuhn, Phys. Rev. B 83, 094303 (2011).

[40] A. M. Barth, A. Vagov, and V. M. Axt, Phys. Rev. B 94, 125439 (2016).

[41] A. J. Leggett, S. Chakravarty, A. T. Dorsey, M. P. A. Fisher, A. Garg, and W. Zwerger, Rev. Mod. Phys. 59, 1 (1987).

[42] E. Sim and N. Makri, The Journal of Physical Chemistry B 101, 5446 (1997).

[43] P. Nalbach and M. Thorwart, Phys. Rev. Lett. 103, 220401 (2009).

[44] A. M. Barth, S. Lüker, A. Vagov, D. E. Reiter, T. Kuhn, and V. M. Axt, Phys. Rev. B 94, 045306 (2016).

[45] M. Glässl, A. M. Barth, and V. M. Axt, Phys. Rev. Lett. 110, 147401 (2013).

[46] M. Glässl and V. M. Axt, Phys. Rev. B 86, 245306 (2012). 
[47] E. Sim, The Journal of Chemical Physics 115, 4450 (2001).

[48] N. Makri, The Journal of Chemical Physics 141, 134117 (2014).

[49] L. Magazzù, D. Valenti, A. Carollo, and B. Spagnolo, Entropy 17, 2341 (2015).

[50] M. Glässl, L. Sörgel, A. Vagov, M. D. Croitoru, T. Kuhn, and V. M. Axt, Phys. Rev. B 86, 035319 (2012).

[51] L. Besombes, Y. Léger, L. Maingault, D. Ferrand, H. Mariette, and J. Cibert, Phys. Rev. Lett. 93, 207403 (2004).

[52] A. Kudelski, A. Lemaître, A. Miard, P. Voisin, T. C. M. Graham, R. J. Warburton, and O. Krebs, Phys. Rev.
Lett. 99, 247209 (2007).

[53] D. E. Reiter, T. Kuhn, and V. M. Axt, Phys. Rev. Lett. 102, 177403 (2009).

[54] A. Blais, R.-S. Huang, A. Wallraff, S. M. Girvin, and R. J. Schoelkopf, Phys. Rev. A 69, 062320 (2004).

[55] Y. Makhlin, G. Schön, and A. Shnirman, Rev. Mod. Phys. 73, 357 (2001).

[56] D. P. S. McCutcheon and A. Nazir, New Journal of Physics 12, 113042 (2010).

[57] B. R. Mollow, Phys. Rev. 188, 1969 (1969). 